\title{
Pediatric obesity: Causes, symptoms, prevention and treatment (Review)
}

\author{
SHUMEI XU and YING XUE \\ Department of Endocrinology, Xuzhou Children's Hospital, Xuzhou, Jiangsu 221002, P.R. China
}

Received September 28, 2015; Accepted November 10, 2015

DOI: 10.3892/etm.2015.2853

\begin{abstract}
Pediatric or childhood obesity is the most prevalent nutritional disorder among children and adolescents worldwide. Approximately 43 million individuals are obese, $21-24 \%$ children and adolescents are overweight, and 16-18\% of individuals have abdominal obesity. The prevalence of obesity is highest among specific ethnic groups. Obesity increases the risk of heart diseases in children and adults. Childhood obesity predisposes the individual to insulin resistance and type 2 diabetes, hypertension, hyperlipidemia, liver and kidney diseases and causes reproductive dysfunction in adults. Obesity in children is a major health concern of the developed world. The National Health and Nutrition Examination Survey has reported that the prevalence of obesity is on the increase in all the pediatric age groups, in males and females, and in various ethnic and racial groups. Factors, such as eating habits, genetics, environment, metabolism, and lifestyle play an important role in the development of obesity. Over $90 \%$ of obesity cases are idiopathic and less than $10 \%$ are associated with genetic and hormonal causes. Obesity occurs when the body consumes more calories than it burns, through overeating and underexercising. The symptoms of obesity include breathing disorders, sleep apnea, chronic obstructive pulmonary disease, certain types of cancer such as prostate, bowel, breast and uterine, coronary heart disease, diabetes (type 2 in children), depression, liver and gallbladder problems, gastro-esophageal reflux disease, high blood pressure, high cholesterol, stroke, and joint diseases such as osteoarthritis, pain in knees and lower back. Environmental, behavioral such as consumption of convenience foods, genetic, and family factors contribute to pediatric obesity. Obesity can be countered through lower calorie consumption, weight loss and diet programs, as well as increased physical activity. A number of endogenous molecules including leptin, hypothalamic melanocortin 4 receptor, and mitochondrial uncoupling
\end{abstract}

Correspondence to: Dr Ying Xue, Department of Endocrinology, Xuzhou Children's Hospital, 18 Sudibei Road, Xuzhou, Jiangsu 221002, P.R. China

E-mail: xueying96@yeah.net

Key words: pediatric obesity, nutritional disorder, insulin resistance, type 2 diabetes, hypertension, hyperlipidemia proteins, are known to affect body weight. These molecules serve as potential targets for the pharmacological manipulation of obesity. Sibutramine and orlistat are primariliy used for the treatment of adult obesity, which produces modest weight loss, of 3-8\% compared to placebo. For children and obese adolescents, metformin is used in the case of insulin resistance and hyperinsulinemia. Octreotide is used for hypothalamic obesity. Bariatric surgery is performed for the treatment of severe childhood obesity. The causes, symptoms, prevention and treatment of pediatric obesity are described in the present review.

\section{Contents}

1. Introduction

2. Causes of childhood obesity

3. Symptoms and complications of obesity

4. Genetic, behavioral and environmental factors of obesity

5. Complications of childhood obesity

6. Convenience foods and obesity

7. Family factors and obesity

8. Physical activity and obesity

9. Prevention and treatment of pediatric obesity

10. Pharmacological and surgical treatment of pediatric obesity

\section{Introduction}

Pediatric or childhood obesity is a growing global epidemic that requires attention due to the burden placed on the healthcare system for children and adults (1). Consumption of fatty foods and a high sugar diet, as well as tobacco smoking, and no exercise qualify as the main reasons for obesity among children and adults. Obesity affects $34 \%$ of children in the USA, and is considered a top public health concern due to the high level of morbidity and mortality. Medical costs for obesity care have escalated, which accounted for $40 \%$ of the healthcare budget in 2006, with billions of dollars being spent on health care annually (1). For the pediatric health care delivery system, expenses were $\$ 179$ per year higher in obese children versus children with a normal body mass index (BMI) (?). Pediatric obesity affects all the organs in the body and has an increasing 
prevalence in young diabetic children. Childhood obesity is connected with an increased risk of various diseases such as diabetes, cardiovascular, stroke, certain types of cancer later in life, social problems and depression among youth (2). As an increasing number of children are becoming overweight, health professionals need to search for effective methods for the prevention and treatment of obesity. In the past 30 years, pediatric obesity has more than doubled in children and tripled in adolescents worldwide (3). Among children aged 6-11 years, the rate of obesity has increased from $7 \%$ in 1980 to $18 \%$ in 2010. This trend was observed among teenagers aged 12-19 years where the rate of obesity increased from 5 to $18 \%$ during the same period of time. Obesity may be defined as having excess body fat and can be determined by the BMI percentage using a growth chart (3). Individuals with an $85-95 \%$ BMI are classified as overweight and those with $95 \%$ BMI are classified as obese. Growth charts can be used to monitor growth over time. These charts are divided by percentile curves to demonstrate the distribution of body measurements. The growth charts are provided by the Centers for Disease Control and Prevention and can be used for obesity.

\section{Causes of childhood obesity}

Obesity occurs when the body consumes more calories than it burns through overeating and underexercising. Childhood obesity is caused by excessive food consumption and drinking of high-calorie sweetened beverages, no exercise or physical activity, as well as genetic factors. The body weight is regulated by various physiological mechanisms that maintain the balance between energy intake and energy expenditure. These regulatory systems under normal conditions, e.g., a positive energy balance of only $500 \mathrm{~kJ}$ (120 kcal) per day (approximately one serving of sugar-sweetened soft drink) would produce a $50 \mathrm{~kg}$ increase in body mass over 10 years. Therefore, factors that can raise energy intake or decrease energy expenditure cause obesity in the long term. Genetic factors have a significant impact on individual predisposition, but other factors of behavior and environment may also play a role in childhood obesity.

\section{Symptoms and complications of obesity}

Symptoms and complications of obesity as well as health risks include, breathing disorder such as sleep apnea and chronic obstructive pulmonary disease, and certain types of cancer such as prostate, bowel in men, breast and uterine cancer in women, coronary heart disease, diabetes (type 2 in children), depression, liver and gall bladder problems, gastro-esophageal reflux disease, high blood pressure, high cholesterol, stroke, and diseases of joints such as osteoarthritis, pain in knees and lower back (4). Obese individuals are likely to have more medical and health problems. Additionally, they are likely to have an accumulation of abdominal fat.

\section{Genetic, behavioral and environmental factors of obesity}

A number of factors contribute to pediatric or childhood obesity. These can be divided into genetic, behavioral and environmental factors. Genetic factors stem from the genes of parents, frequently leading to children becoming overweight (5). Behavioral factors include food consumption and drinking of high-calorie sugar-sweetened beverages that are of low nutritional value, which are readily available for children. Consumption of the latter in particular has been associated with obesity (5).

Lack of physical activity also contributes to obesity. Children spend a large amount of time using technology such as cell phones, television, computers or video games. On average, children of 8-18 years spend $7.5 \mathrm{~h}$ per day using these gadgets and do not participate in physical activities and active play (5). Concerning environmental factors, the home, school, and community environments all play an important role in a child's development and maintenance of a healthy diet and involvement in physical activity. The majority of young children are enrolled in schools providing an optimum environment whose aim is to reinforce healthy eating and physical activity behaviors. When not in school, experiences in a child care or home setting continue to shape dietary and physical activity behaviors. Another crucial factor is the communities in which children reside as they provide opportunities for physical activity and access to affordable and healthy foods.

Obese children are more likely to become overweight in adulthood than healthy-weight children. This increase in weight constitutes a health risk later in life when, as adults, they present with problems such as heart disease, diabetes (type 2), stroke, various types of cancer and osteoarthritis (5). In addition, obese children are more likely to have cardiovascular risk factors such as high cholesterol or high blood pressure, and are more likely to have pre-diabetes, which places them at a high risk for developing diabetes later in life (5). Thus maintaining a healthy diet and involvement in physical activity are important for preventing obesity and other diseases.

The dietary and physical activity behaviors of children and adolescents are affected by communities, schools, child care settings, faith-based institutions, government agencies, families, the media, as well as the food and beverage industries. the impact of the last factor led to the US Better Business Bureau established the Children's Food and Beverage Advertising Initiative (5). This initiative is a voluntary self-regulated program that includes many of the largest food and beverage companies in the USA. The purpose of this initiative is to encourage the choice of following a healthy diet and healthy lifestyle among children by shifting the types of food and beverages advertised to children under the age of 12 years. Schools play an important role in establishing an environment that supports healthy lifestyle habits. Policies within schools can be established to encourage healthy eating and regular physical activity for children. Schools have the opportunity to both educate students about these behaviors and provide an opportunity for the practice thereof. In an effort to increase the number of fruits and vegetables served in schools, programs such as 'Let's Move Salad Bars to Schools and the National Farm to School Network' have been introduced (6). In response to the childhood obesity epidemic, the 'Let's Move Salad Bars to Schools' campaign was established by Michelle Obama, the First Lady of the USA (6). The treatment for childhood obesity depends on the age of the child and existing medical conditions. 
Treatments for obesity include changes to the diet and level of physical activity of the child. The purpose of the treatment for obesity involves weight maintenance as opposed to weight loss. The growth of children primarily is measured as height, and not weight, leading to a decrease of BMI per age point, into a healthier range. However, in the case of obesity, depending on the health conditions of children, treatment may involve a multidisciplinary team including the family physician, dietitian, counselor or physical therapist. Specific plans for diet and physical activity have been developed where general guidance is ineffective. In most cases, a low-calorie diet and weight loss drugs may be administered to control individuals becoming overweight or exhibiting abdominal obesity.

A review on childhood obesity by Pulgarón has focused on the increased risk for physical and psychological comorbidities (4). Approximately 43 million children worldwide are estimated to be encountering a problem of obesity, and this number is on the increase. An initial search of the terms obesity and comorbidity yielded $>5,000$ published articles. The limits were set to include studies on children and adolescents that were published in journals between 2002 and 2012. These limits narrowed the search to 938 articles, 79 of which have been included in the present review. The major medical comorbidities associated with childhood obesity in the current literature are metabolic risk factors, asthma, and dental health quality (6). Major psychological comorbidities include internalizing and externalizing disorders, attention deficit hyperactivity disorder, and problems with sleep $(6,7)$. Notably, some of the results were based on self-report diagnoses rather than chart reviews or diagnosis by a physician. The decreased health-related quality of life as a comorbidity of obesity in children has also been reported.

Han et al (8) identified an increase in the occurrence of type 2 diabetes in children, which is believed to be a consequence of obesity. Progress has been made in understanding the physiology and genetics of appetite control, which have been useful in determining the causes of some rare obesity syndromes. Calorie intake and physical activity or lack thereof should be reassessed and the quantification improved at a population level since the majority of children lead a sedentary lifestyle. The current recommended calorie prescriptions may be too conservative to impact the so-called energy gap. The dietary recommendation and daily calorie intake for healthy children is shown by the American Heart Association (AHA, May 14, 2015) as follows: Maintain total fat intake between 30 and $35 \%$ of calories for children aged 2-3 years, and between 25 and 35\% of calories for children and adolescents aged 4-18 years, with most fats coming from sources of polyunsaturated and monounsaturated fatty acids, such as fish, nuts and vegetable oils. Children should consume sufficient carbohydrates, fruits and vegetables but not in large amounts. The daily recommended calories needed for children aged 1 year is $900 \mathrm{cal} /$ day and 1,800 calories for a 14- to 18-year-old girl and 2,200 calories for a 14- to 18-year-old boy. The recommended grain intake ranges from 2 ounces/day for a one-year-old to 7 ounces/day for a 14- to 18-year-old boy. Consumption of low-fat dairy products is recommended to maintain a healthy body weight and height. Children are required to be physically active and exercise for $\geq 60 \mathrm{~min}$ per day.
The quality of new research into obesity prevention and treatment has been markedly improved. However, high-quality multicentre trials with long-term follow-up studies are required. Prevention and treatment approaches to increase energy expenditure and decrease energy intake should also be performed. Previous findings have suggested that the increase in the prevalence of childhood obesity may be abating and increased efforts should be made to further investigate obesity in children (6). A literature review of family in pediatric obesity management has been performed (6). Obese children and adolescents are considered at increased risk for multiple medical comorbidities, as well as psychosocial and behavioral difficulties (9). Interventions aimed at childhood obesity include prevention and treatment, which should be ameliorated in order to become useful and beneficial in the clinical setting.

The aim of this review was to examine the effectiveness of family-based interventions in obese pediatric subjects and to determine the specific components of family-based programs that are of particular significance when treating obese children. In addition, to develop a completely interactive obesity model, more focus is needed on the specific methods that can be used in evidence-based programs. Ebbeling et al have reported childhood obesity as a public health crisis, and common sense cure and several complications of childhood obesity have been described (10).

\section{Complications of childhood obesity}

Childhood obesity is a multisystem disease with potentially adverse consequences and several complications that require the attention of health professionals. Obesity in childhood causes hypertension, dyslipidemia, chronic inflammation, increased blood clotting tendency, endothelial dysfunction, and hyperinsulinemia (11-15). This clustering can cause cardiovascular disease risk factors, known as the insulin resistance syndrome, which has been identified in children as young as 5 years of age (16). For adolescents and young adults who succumbed to trauma, the presence of cardiovascular disease risk factors correlated with asymptomatic coronary atherosclerosis, and lesions were more advanced in obese individuals $(17,18)$. In a British cohort study (19), being overweight during childhood increased the risk of death from ischemic heart disease in adulthood 2-fold for individuals $>57$ years.

Type 2 diabetes, in adolescence, now accounts for almost $50 \%$ of all new diagnoses of diabetes in certain populations (20). There is a health concern for prediabetic state, consisting of glucose intolerance and insulin resistance, which seems to be highly prevalent among severely obese children irrespective of ethnic group, and even before formal diagnostic criteria for diabetes have been met (21). The emergence of type 2 diabetes in children is a negative development, in view of the macro-vascular (heart disease, stroke, limb amputation) and micro-vascular (kidney failure, blindness) sequelae. Frequent pulmonary complications include sleep-disordered breathing (sleep apnea) (22), asthma (23) and exercise intolerance (24). The development of asthma or exercise intolerance in an obese child are likely to limit physical activity and cause health problems. 


\section{Convenience foods and obesity}

The rise in the consumption of convenience foods by children and adolescents in many nations has a particular relevance to the childhood obesity epidemic (10). Convenience foods incorporate all of the potentially adverse dietary factors, including saturated and trans fats, a high glycemic index, high energy density and increasingly large portions of food. Convenience foods are usually low in fiber, micronutrients and antioxidants and contain dietary components that affect risk of heart diseases, cardiovascular events and diabetes in children. There is an association between the consumption of convenience foods and total energy intake or body weight in adolescents and adults (25). Adolescent females who consumed convenience foods four times a week or more consume $\sim 770-1095 \mathrm{~kJ}(\sim 185-260 \mathrm{kcal})$ per day more than those who did not consume such food (25). A large meal of this type such as that found at the majority of convenience food outlets, i.e., double cheeseburger, French fries, soft drink and a dessert, is likely to contain 9,200 kJ (2,200 kcal), which, at $350 \mathrm{~kJ}$ (85 kcal) per mile, would require a full marathon to eliminate the body fat.

\section{Family factors and obesity}

Parent-child interactions and the home environment may affect behavior associated with risk of obesity. Family life has changed over the past two decades, with lifestyle habits tending towards dining out and greater access to various forms of technology, including television, than previous years. Owing to larger portions of energy dense foods being served at restaurants, energy intake is greater when meals are consumed in restaurants compared to homemade meals (26). In addition, unlimited access to television has increased the period spent watching by 38 min per day (27). By contrast, family meals seem to decrease time spent on television watching and improve the quality of the diet as less saturated and trans fat, less fried food, lower glycemic load, more fiber, fewer sweetened beverages, and more fruits and vegetables are consumed (28). Moreover, social support from parents and other community members correlates strongly with participation in physical activity (29). Thus, psychosocial factors are crucial to dietary and physical activity behaviors that affect energy balance as children who suffer from neglect, depression, and other associated issues are at an increased risk of childhood obesity, which eventually may result in overweight adults.

\section{Physical activity and obesity}

Williams et al studied the cardiovascular health in childhood obesity and other diseases such as atherosclerosis and hypertension in young people in the USA (30). Health benefits were associated with a physically active lifestyle in children, including a weight control program, physical exercise for obese individuals, low blood pressure, modest consumption of fatty foods, and high-calorie drinks or beverages. Engaging in physical activity likely increases life expectancy and decreases the risk of heart diseases in children and younger individuals. Attaining healthy levels of physical fitness requires a regular exercise program of 4-5 times per week for 30-60 min (30). Such exercise may include walking, cycling, and sports activities versus sedentary lifestyle activities, which are primarily technology-oriented. Lack of safe outdoor playgrounds in parks and schools for children in cities also limits children's ability to engage in active physical play or recreational sports (30). Thus, a physical fitness program is essential for the pediatric health care of children.

\section{Prevention and treatment of pediatric obesity}

The prevention and treatment of pediatric obesity can be achieved by less food consumption and increased physical activity. Of note, however, is that long-term weight loss and diet programs have proven exceedingly difficult to achieve. The National Institutes of Health, USA consensus statement indicates that the adults who remain in conventional weight loss programs can realistically expect a maximum weight loss of only 10\% (a small fraction of excess adiposity) (30). Approximately $50 \%$ of this modest weight loss is regained within a year, and almost all of the weight is regained within 5 years (31). The relative intellectual and psychological immaturity of children compared with adults, and their susceptibility to peer pressure present additional practical problems to the successful treatment of childhood obesity. The majority of efforts to reduce obesity in children have used family- or school-based approaches, although pharmacological and surgical treatments are also available (32).

\section{Pharmacological and surgical treatment of pediatric obesity}

New research on the elucidation of the physiological basis of bodyweight regulation, and sequencing of the human genome have provided an opportunity to develop new anti-obesity agents. An increasing number of endogenous molecules such as leptin, hypothalamic melanocortin 4 receptor, and mitochondrial uncoupling proteins, known to affect body weight serve as potential targets for the pharmacological manipulation of obesity (33). The majority of drugs used for the treatment of obesity over the previous century, from thyroid extract in the 1890 s to Fen-Phen in the 1990s showed potentially life-threatening complications. Drugs do not produce permanent changes in physiology or behavior, since these are effective provided they are taken, which raises the specter of life-long treatment. Sibutramine and orlistat, which are frequently used for the treatment of adult obesity, produce modest weight loss ranging from $\sim 3$ to $8 \%$ compared to placebo (33). Four experimental drugs have produced weight loss, as identified in small-scale studies that involved children with special conditions. These drugs are, Metformin, used in obese adolescents with insulin resistance and hyperinsulinemia (34), octreotide, used for hypothalamic obesity (35), growth hormone in children with Prader-Willi syndrome (36), and leptin for congenital leptin deficiency (37). Bariatric surgery is used for the treatment of severe obesity in childhood (38). The Roux-en-Y gastric bypass has been reported for significant weight loss (39). However, serious complications of this procedure can result in perioperative mortality, wound dehiscence, bowel obstruc- 
tion, gastrointestinal bleeding, cholelithiasis, infection, and chronic nutritional deficiencies. This approach constitutes a last resort for the treatment of severely obese adolescents as emphasized by Strauss et al (39).

In conclusion, the worldwide prevalence of pediatric obesity has increased several-fold in recent years. Obese children develop serious health, medical and psychosocial complications, and are at an increased risk of adult morbidity and mortality. The increase in the prevalence and severity of obesity in children, with its most serious complication, type 2 diabetes raises the specter of myocardial infarction which is a pediatric heart disease. This health crisis among obese children and young adults requires greater attention and government funding for the research of children diseases. New dietary, physical activity, behavioral, environmental, and pharmacological approaches can be used for the prevention and treatment of pediatric obesity. Improved reimbursement is required for effective family- and school-based health programs. Since the obesity epidemic is not caused by inherent biological defects, the increase in research funding and health care for the treatment of pediatric obesity may not serve as a solution to the problem without fundamental measures to effectively detoxify the environment. In the long term, financial investment is imperative for the healthy lifestyle of children and adults in modern society. Obesity and diabetes are growing challenges in the developing world (40). Changes in lifestyle that lead to weight loss reduce the incidence of diabetes and hypertension. Prevention of obesity, diabetes, and hypertension may require fundamental social and political changes. Public health initiatives are required to make affordable, healthy foods available, and initiatives in education and community planning are necessary to encourage and facilitate exercise on a daily basis. The World Health Organization has issued objectives for developing countries regarding school meals and healthy lifestyles. Countries, such as Brazil, China and India have initiated monitoring programs associated with obesity and nutrition (41). Since these programs are in their infancy, the cost of their implementation and effectiveness remain to be determined. However, the general consensus is that appropriate and acceptable strategies should be implemented to control pediatric obesity, diabetes and hypertension.

\section{References}

1. Rome ES: Obesity prevention and treatment. Pediatr Rev 32 363-372, quiz 373, 2011.

2. Childhood Obesity: Assessment, Prevention \& Treatment. Childhood Obesity: Overview, 2015.

3. Centers for Disease Control and Prevention: BMI guidelines with linkstothe growthcharts.cdc.gov/nccdphp/dnpa/bmi/bmi-for-age. html.

4. Rettner R. Obesity: causes, complications and treatment. Live Sc. Feb, 3, 2015.

5. Hill JO, Peters JC. Environmental contribution to the obesity epidemic. Science 280: 1371-1374,1998.

6. Harris DM, Seymour J, Grummer-Strawn L, Cooper A,Collins B, DiSograL, Marshall A and Evans N: Let's move salad bars to schools: a public-private partnership to increase student fruit and vegetable consumption. Child Obes: 8, 294-297, 2012. doi: 10.1089/chi.2012.0094

7. Pulgarón ER: Childhood obesity: a review of increased risk for physical and psychological comorbidities. Clin Ther 35: A18-A32, 2013.

8. Han JC, Lawlor DA and Kimm SYS: Childhood obesity. Lancet 375: 1737-1748, 2010.
9. Nowicka P and Flodmark CE: Family in pediatric obesity management: a literature review. Int J Pediatr Obes 3 (Suppl 1): 44-50, 2008.

10. Ebbeling CB, Pawlak DB and Ludwig DS: Childhood obesity: Public-health crisis, common sense cure. Lancet 360: 473-482, 2002.

11. Freedman DS, Dietz WH, Srinivasan SR and Berenson GS: The relation of overweight to cardiovascular risk factors among children and adolescents: The Bogalusa Heart Study. Pediatrics 103: 1175-1182, 1999.

12. Ford ES, Galuska DA, Gillespie C, Will JC, Giles WH and Dietz WH: C-reactive protein and body mass index in children: Findings from the Third National Health and Nutrition Examination Survey, 1988-1994. J Pediatr 138: 486-492, 2001.

13. Ferguson MA, Gutin B, Owens S, Litaker M, Tracy RP and Allison J: Fat distribution and hemostatic measures in obese children. Am J Clin Nutr 67: 1136-1140, 1998.

14. Tounian P, Aggoun Y, Dubern B, Varille V, Guy-Grand B, Sidi D, Girardet JP and Bonnet D: Presence of increased stiffness of the common carotid artery and endothelial dysfunction in severely obese children: a prospective study. Lancet 358: 1400-1404, 2001.

15. Srinivasan SR, Myers L and Berenson GS: Predictability of childhood adiposity and insulin for developing insulin resistance syndrome (syndrome $\mathrm{X}$ ) in young adulthood: the Bogalusa Heart Study. Diabetes 51: 204-209, 2002.

16. Young-Hyman D, Schlundt DG, Herman L, De Luca F and Counts D: Evaluation of the insulin resistance syndrome in 5- to 10-year-old overweight/obese African-American children. Diabetes Care 24: 1359-1364, 2001.

17. Strong JP, Malcom GT, McMahan CA, Tracy RE, Newman WP III, Herderick EE and Cornhill JF: Prevalence and extent of atherosclerosis in adolescents and young adults: implications for prevention from the Pathobiological Determinants of Atherosclerosis in Youth Study. JAMA 281: 727-735, 1999.

18. McGill HC Jr, McMahan CA,Zieske AW, Tracy RE, Malcom GT, Herderick EE and Strong JP: Association of Coronary Heart Disease Risk Factors with microscopic qualities of coronary atherosclerosis in youth. Circulation 102: 374-379, 2000.

19. Gunnell DJ, Frankel SJ, Nanchahal K, Peters TJ and Davey Smith G: Childhood obesity and adult cardiovascular mortality: a 57-y follow-up study based on the Boyd Orr cohort. Am J Clin Nutr 67: 1111-1118, 1998.

20. Fagot-Campagna A, Pettitt DJ, Engelgau MM, Burrows NR, Geiss LS, Valdez R, Beckles GL, Saaddine J, Gregg EW, Williamson DF, et al: Type 2 diabetes among North American children and adolescents: an epidemiologic review and a public health perspective. J Pediatr 136: 664-672, 2000.

21. Sinha R, Fisch G, Teague B, Tamborlane WV, Banyas B, Allen K, Savoye M, Rieger V, Taksali S, Barbetta G, et al: Prevalence of impaired glucose tolerance among children and adolescents with marked obesity. N Engl J Med 346: 802-810, 2002.

22. Redline S, Tishler PV, Schluchter M, Aylor J, Clark K and Graham G: Risk factors for sleep-disordered breathing in children. associations with obesity, race, and respiratory problems. Am J Respir Crit Care Med 159: 1527-1532, 1999.

23. Figueroa-Muñoz JI, Chinn S and Rona RJ: Association between obesity and asthma in 4-11 year old children in the UK. Thorax 56: 133-137, 2001.

24. Reybrouck T, Mertens L, Schepers D, Vinckx J and Gewillig M: Assessment of cardiorespiratory exercise function in obese children and adolescents by body mass-independent parameters. Eur J Appl Physiol Occup Physiol 75: 478-483, 1997.

25. McNutt SW, Hu Y, Schreiber GB, Crawford PB, Obarzanek E and Mellin L: A longitudinal study of the dietary practices of black and white girls 9 and 10 years old at enrollment: the NHLBI Growth and Health Study. J Adolesc Health 20: 27-37, 1997.

26. Zoumas-Morse C, Rock CL, Sobo EJ and Neuhouser ML: Children's patterns of macronutrient intake and associations with restaurant and home eating. J Am Diet Assoc 101: 923-925, 2001.

27. Wiecha JL, Sobol AM, Peterson KE and Gortmaker SL: Household television access: associations with screen time, reading, and homework among youth. Ambul Pediatr 1: 244-251, 2001.

28. Gillman MW, Rifas-Shiman SL, Frazier AL, Rockett HR, Camargo CA Jr, Field AE, Berkey CS and Colditz GA: Family dinner and diet quality among older children and adolescents. Arch Fam Med 9: 235-240, 2000. 
29. Sallis JF, Prochaska JJ and Taylor WC: A review of correlates of physical activity of children and adolescents. Med Sci Sports Exerc 32: 963-975, 2000.

30. Williams CL, Hayman LL, Daniels SR, Robinson TN, Steinberger J, Paridon S and Bazzarre T: Cardiovascular health in childhood: A statement for health professionals from the Committee on Atherosclerosis, Hypertension, and Obesity in the Young (AHOY) of the Council on Cardiovascular Disease in the Young, American Heart Association. Circulation 106: 143-160, 2002.

31. No authors listed: Methods for voluntary weight loss and control. NIH Technology Assessment Conference Panel. Consensus Development Conference, 30 March to 1 April 1992. Ann Intern Med 119: 764-770, 1993.

32. Barlow SE and Dietz WH: Obesity evaluation and treatment: Expert Committee recommendations. The Maternal and Child Health Bureau, Health Resources and Services Administration and the Department of Health and Human Services. Pediatrics 102: E29, 1998.

33. Glazer G: Long-term pharmacotherapy of obesity 2000: A review of efficacy and safety. Arch Intern Med 161: 1814-1824, 2001.

34. Freemark M and Bursey D: The effects of metformin on body mass index and glucose tolerance in obese adolescents with fasting hyperinsulinemia and a family history of type 2 diabetes. Pediatrics 107: E55, 2001.
35. Lustig RH, Rose SR, Burghen GA, Velasquez-Mieyer P, Broome DC, Smith K, Li H, Hudson MM, Heideman RL and Kun LE: Hypothalamic obesity caused by cranial insult in children: Altered glucose and insulin dynamics and reversal by a somatostatin agonist. J Pediatr 135: 162-168, 1999.

36. Myers SE, Carrel AL, Whitman BY and Allen DB: Sustained benefit after 2 years of growth hormone on body composition, fat utilization, physical strength and agility, and growth in Prader-Willi syndrome. J Pediatr 137: 42-49, 2000.

37. Farooqi IS, Jebb SA, Langmack G, Lawrence E, Cheetham $\mathrm{CH}$, Prentice AM, Hughes IA, McCamish MA and O'Rahilly S: Effects of recombinant leptin therapy in a child with congenital leptin deficiency. N Engl J Med 341: 879-884, 1999.

38. Yanovski JA: Intensive therapies for pediatric obesity. Pediatr Clin North Am 48: 1041-1053, 2001.

39. Strauss RS, Bradley LJ and Brolin RE: Gastric bypass surgery in adolescents with morbid obesity. J Pediatr 138: 499-504, 2001.

40. Hossain P, Kawar B and El Nahas M: Obesity and diabetes in the developing world - a growing challenge. N Engl J Med 356: 213-215, 2007

41. Daniels SR, Arnett DK, Eckel RH, et al: Overweight in children and adolescents: pathophysiology, consequences, prevention, and treatment. Circulation 111: 1999-2002, 2005. 